\title{
ON THE COMPLETENESS OF ORBITS OF A POMMIEZ OPERATOR IN WEIGHTED (LF)-SPACES OF ENTIRE FUNCTIONS
}

\author{
Olga A. Ivanova, Sergej N. Melikhov \\ Vorovich Institute of Mathematics, Mechanics and Computer Sciences, \\ Southern Federal University \\ Mil'chakova st. 8a, 344090 Rostov on Don, Russia \\ neo_ivolga@mail.ru \\ Vorovich Institute of Mathematics, Mechanics and Computer Sciences, \\ Southern Federal University \\ Mil'chakova st. 8a, 344090 Rostov on Don, Russia \\ and Southern Mathematical Institute of Vladikavkaz Science Center \\ of the Russ. Acad. Sci. \\ Markus st. 22, 362027 Vladikavkaz, Russia \\ melih@math.rsu.ru
}

Keywords: cyclic element, Pommiez operator, (LF)-space of entire functions 2010 MSC: Primary 47A16, Secondary 47B38, 46E10

\begin{abstract}
We describe cyclic vectors for a Pommiez operator on a weighted (LF)-space $E$ of entire functions. The full description is obtained where $E$ is the Laplace transform of the strong dual of the space of all germs of holomorphic functions on a convex locally closed set in the complex plane.
\end{abstract}

\section{INTRODUCTION}

In [19]-21] Pommiez studied consecutive remainders for the Taylor series of analytic functions on the disk zentered at zero. In [22] generalized Newton series expansions for analytic functions were investigated. In [19]-[22] the following difference operator was used:

$$
D_{z}(f)(t):= \begin{cases}\frac{f(t)-f(z)}{t-z}, & t \neq z, \\ f^{\prime}(z), & t=z .\end{cases}
$$

After the papers [19]-22, it has become to refer to the operators $D_{z}$ as Pommiez operators. The operator $D_{0}$ is called also the backward shift operator. In [6] an interpolating functional was studied that plays an important role in the theory of exponential series and convolution equations for analytic functions. This functional is an abstract version of Leont'ev's interpolating function. It is defined with the help of a Pommiez operator $D_{0, g_{0}}$ acting continuously and linearly in a weighted (LF)-space $E$ of entire functions. The operator $D_{0, g_{0}}$ is defined by

$$
D_{0, g_{0}}(f)(t):= \begin{cases}\frac{f(t)-g_{0}(t) f(0)}{t}, & t \neq 0, \\ f^{\prime}(0)-g_{0}^{\prime}(0) f(0), & t=0 .\end{cases}
$$

Here $g_{0}$ is a fixed function in $E$ such that $g_{0}(0)=1$. If $g_{0}$ is identically equal to 1 , the operator $D_{0, g_{0}}$ coincides with the operator $D_{0}$. If $E$ does not contain the function $g_{0} \equiv 1$, then the standard operator $D_{0}$ may fail to act in $E$. Hence the passage to 
a function $g_{0} \in E$ with $g_{0}(0)=1$ proves to be quite natural. Tkachenko [23], [24] investigated the operator of generalized integration, which is the dual map of $D_{0, g_{0}}$ in the case $g_{0}=e^{P}$ where $P$ is a polynomial. This operator $D_{0, g_{0}}$ acts in a weighted (LB)space of entire functions whose growth is determined with the help of a $\rho$-trigonometric convex function $(\rho>0)$.

In the present article we study cyclic vectors for $D_{0, g_{0}}$ in $E$. A vector $x$ in a locally convex space $F$ is called cyclic for a continuous linear operator $A$ in $F$ provided the linear span of the orbit $\left\{A^{n}(x): n \geq 0\right\}$ is dense in $F$. Earlier similar results were obtained in following situations. Khaplanov [10] found a sufficient cyclicity condition for a function for the operator $D_{0}$ in the space of all analytic functions on the disk $|z|<R$. Kaz'min [9] proved cyclicity criteria for a function for $D_{0}$ in the Fréchet space $H(G)$ of all analytic functions on a simply connected domain $G$ in $\mathbb{C}$ containing zero. N. Linchuk [14] investigated cyclic vectors for the operator $D_{0}$ in $H(G)$ for a finitely connected domain $G$ in $\mathbb{C}$. Yu. Linchuk [15] studied cyclic vectors of some "onedimensional variation" of $D_{0}$ in $H(G)$. This operator is the operator $D_{0, g_{0}}$ for some function $g_{0} \in H(G)$ (see [7]). In [7] cyclic vectors were described for $D_{0, g_{0}}$ in $H(G)$ without additional assumptions of the paper [15]. Douglas, Shapiro and Shields [4] investigated cyclic vectors and invariant subspaces for the backward shift operator $D_{0}$ in the Hardy space $H^{2}$ on the unit disk. Cyclic elements for the generalized backward shift operator were described also by Godefroy and Shapiro [5].

In $\S 1$ we introduce the space $E$ and operators of our interest. In $\S 2$ we prove abstract sufficient and necessary conditions for the completeness of the system $\left\{D_{0, g_{0}}^{n}(f): n \geq 0\right\}$ in $E$. In $\S 3$ we apply obtained results to the space $E$, which is the realization (with the help of the Laplace transform) of the strong dual of the space $H(Q)$ of all germs of analytic functions on a convex locally closed set $Q \subset \mathbb{C}$. Main result here is Theorem 19 , in which the full description of cyclic vectors for $D_{0, g_{0}}$ in $E$ is obtained. In the proof of Theorem 19 we use essentially Leont'ev's interpolating function. As a consequence we characterize proper closed $D_{0, g_{0}}$-invariant subspaces of such space $E$ in the case that $g_{0}$ has no zeros and proper closed ideals in the algebra $(H(Q)$, *) where $*$ is the Duhamel product in $H(Q)$.

\section{AN AUXILIARY INFORMATION}

In [6], 8] a Pommiez operator associated with a function $g_{0}$ in a weighted $(L F)$-space of entire functions was investigated. We mention a necessary information from [6], [8]. For a continuous function $v: \mathbb{C} \rightarrow \mathbb{R}$, for a function $f: \mathbb{C} \rightarrow \mathbb{C}$ we put

$$
p_{v}(f):=\sup _{z \in \mathbb{C}} \frac{|f(z)|}{\exp v(z)} .
$$

Let $v_{n, k}: \mathbb{C} \rightarrow \mathbb{R}$ be continuous functions such that

$$
v_{n, k+1} \leq v_{n, k} \leq v_{n+1, k}, n, k \in \mathbb{N} .
$$

Define weighted Fréchet spaces

$$
E_{n}:=\left\{f \in H(\mathbb{C}): p_{v_{n, k}}(f)<+\infty \forall k \in \mathbb{N}\right\}, n \in \mathbb{N} .
$$

Here $H(\mathbb{C})$ is the space of all entire functions on $\mathbb{C}$ with the compact open topology. Note that $E_{n}$ is embedded continuously in $E_{n+1}$ for each $n \in \mathbb{N}$. Put $E:=\bigcup_{n \in \mathbb{N}} E_{n}$ and 
we endow $E$ with the topology of the inductive limit of the sequence of Fréchet spaces $E_{n}, n \in \mathbb{N}$, with respect to embeddings $E_{n}$ in $E$ (see [17, Ch. III, $\left.\S 24\right]$ ): $E:=$ ind $E_{n}$.

In the sequel, we assume that the double sequence $\left(v_{n, k}\right)_{n, k \in \mathbb{N}}$ satisfies the following condition:

$$
\begin{gathered}
\forall n \exists m \forall k \exists s \exists C \geq 0: \\
\sup _{|t-z| \leq 1} v_{n, s}(t)+\ln (1+|z|) \leq \inf _{|t-z| \leq 1} v_{m, k}(t)+C, z \in \mathbb{C} .
\end{gathered}
$$

Then the space $E$ is invariant under differentiation and translation, and for each $n \in \mathbb{N}$ there exists $m \in \mathbb{N}$ such that every bounded set in $E_{n}$ is relatively compact in $E_{m}$ [6, Remark 1]. Moreover, $E$ is invariant under multiplication by the independent variable.

We assume that $E$ contains a function that is not identically zero. Then there exists a function $g_{0} \in E$ such that $g_{0}(0)=1$. For arbitrary function $g_{0} \in E$ with $g_{0}(0)=1$ we define the Pommiez operator associated with $g_{0}$ by

$$
D_{0, g_{0}}(f)(t):=\left\{\begin{array}{cc}
\frac{f(t)-g_{0}(t) f(0)}{t}, & t \neq 0, \\
f^{\prime}(0)-g_{0}^{\prime}(0) f(0), & t=0 .
\end{array}\right.
$$

The operator $D_{0, g_{0}}$ maps linearly and, by the closed graph theorem, continuously $E$ into itself.

For a locally convex space $F$ we denote by $\mathcal{L}(F)$ the space of all continuous linear operators from $F$ into itself.

Following [1], 3], we introduce the shift operator for the Pommiez operator $D_{0, g_{0}}$

$$
T_{z}(f)(t):=\left\{\begin{array}{cc}
\frac{t f(t) g_{0}(z)-z f(z) g_{0}(t)}{t-z}, & t \neq z, \\
z g_{0}(z) f^{\prime}(z)-z f(z) g_{0}^{\prime}(z)+f(z) g_{0}(z), & t=z,
\end{array}\right.
$$

$f \in E$. By [8] $T_{z} \in \mathcal{L}(E)$ for all $z \in \mathbb{C}$.

If $g_{0}=e^{P}$, where $P$ is a polynomial, Tkachenko [24] used the operator $T_{z}$ for the description of operators commuting with an operator $J_{P}$ of generalized integration. The operator $J_{P}$ is the dual map of $D_{0, e^{P}}$ that acts in a weighted (LB)-space of entire functions. The growth of functions of this space is determined with the help a $\rho$ trigonometric convex function $(\rho>0)$.

We mention some auxiliary statements [8, Lemma 7, Lemma 11, Lemma 12] (here Lemma 1, Lemma 3, Lemma 4, respectively). For $z \in \mathbb{C}$ the symbol $\delta_{z}$ denotes the delta-function $\delta_{z}(f):=f(z)$.

1. Lemma. For each integer $n \geq 0$

$$
D_{0, g_{0}}^{n}(f)(z)=\varphi_{n}\left(T_{z}(f)\right), \quad f \in E, z \in \mathbb{C},
$$

where $\varphi_{0}=\delta_{0}$ and for each $n \in \mathbb{N}$ there exist $c_{k, n} \in \mathbb{C}, 0 \leq k \leq n-1$, such that

$$
\varphi_{n}(f)=\frac{1}{n !} f^{(n)}(0)+\sum_{k=0}^{n-1} c_{k, n} f^{(k)}(0), f \in E .
$$

For a locally convex space $F$ by $F^{\prime}$ we denote the topological dual of $F$. By $\tau:=\tau\left(E^{\prime}, E\right)$ we denote the Mackey topology in $E^{\prime}$, that is, the topology of uniform convergence on the family of all absolutely convex $\sigma\left(E, E^{\prime}\right)$-compact subsets of 
$E$ [17, Ch. III, $\S 3]$. Here $\sigma\left(E, E^{\prime}\right)$ is the weak topology in $E$ which is defined by the natural duality between $E$ and $E^{\prime}$.

2. Remark. (a) Since $E$ is embedded continuously in $H(\mathbb{C})$ for each $k \geq 0$ and $t \in \mathbb{C}$ linear functionals $f \mapsto f^{(k)}(t)$ are continuous on $E$. Hence all functionals $\varphi_{n}, n \geq 0$, in Lemma 1 are continuous on $E$.

(b) If $f \in E$ and $\varphi_{n}(f)=0$ for all $n \geq 0$, then $f^{(n)}(0)=0$ for all $n \geq 0$. Hence the function $f$ vanishes on $\mathbb{C}$. Therefore the system $\left\{\varphi_{n}: n \geq 0\right\}$ is complete in $\left(E^{\prime}, \tau\right)$.

For $f \in E$ we put

$$
\widetilde{f}(t, z):=T_{z}(f)(t), t, z \in \mathbb{C} .
$$

The function $\tilde{f}$ is entire on $\mathbb{C}^{2}$.

3. Lemma. If a net $\Psi_{\mu} \in E^{\prime}, \mu \in \Delta$, converges to $\psi \in E^{\prime}$ in $\left(E^{\prime}, \tau\right)$, then for each function $f \in E$

$$
\lim _{\mu \in \Delta}\left(\Psi_{\mu}\right)_{z}(\tilde{f}(\cdot, z))=\psi_{z}(\tilde{f}(\cdot, z)) \text { and } \lim _{\mu \in \Delta}\left(\Psi_{\mu}\right)_{t}(\tilde{f}(t, \cdot))=\psi_{t}(\tilde{f}(t, \cdot)),
$$

where the limits are taken in $E$.

4. Lemma. Let $\varphi_{n}, n \geq 0$, be the functionals defined by Lemma 1. For each entire function $h$ on $\mathbb{C}^{2}$, for all integers $j, k \geq 0$

$$
\left(\varphi_{j}\right)_{z}\left(\left(\varphi_{k}\right)_{t}(h(t, z))\right)=\left(\varphi_{k}\right)_{t}\left(\left(\varphi_{j}\right)_{z}(h(t, z))\right) .
$$

Let $\mathcal{K}\left(D_{0, g_{0}}\right)$ be the set of all operators $B \in \mathcal{L}(E)$ such that $B D_{0, g_{0}}=D_{0, g_{0}} B$ on $E$. By [8, Theorem 15] the following description of $\mathcal{K}\left(D_{0, g_{0}}\right)$ holds.

5. Theorem. (i) If $B \in \mathcal{K}\left(D_{0, g_{0}}\right)$, there exists a unique $l \in E^{\prime}$ such that $B(f)(z)=$ $l\left(T_{z}(f)\right)$ for all $f \in E$ and $z \in \mathbb{C}$.

(ii) For each $l \in E^{\prime}$ the operator $B(f)(z)=l\left(T_{z}(f)\right), f \in E, z \in \mathbb{C}$, belongs to $\mathcal{K}\left(D_{0, g_{0}}\right)$.

\section{Abstract CRIteria of the CyClicity}

In this section we describe cyclic vectors for $D_{0, g_{0}}$ in $E$.

6. Definition. Let $F$ be a locally convex space. An element $x \in F$ is called a cyclic vector for an operator $A \in \mathcal{L}(F)$ if the system $\left\{A^{n}(x): n \geq 0\right\}$ is complete in $F$, that $i s$, the linear span of $\left\{A^{n}(x): n \geq 0\right\}$ is dense in $F$.

For a locally convex space $F$ and an operator $A \in \mathcal{L}(F)$ the $\operatorname{symbol} \mathrm{Cycl}_{F}(A)$ denotes the set of all cyclic vectors of $A$ in $F$. Further $E$ is the space as in $\S 1$. We will write $\operatorname{Cycl}(A)$ instead of $\operatorname{Cycl}_{E}(A)$.

The following assertion is obvious.

7. Lemma. Let $F$ be a locally convex space and $A \in \mathcal{L}(F)$ be surjective. Then the set $\mathrm{Cycl}_{F}(A)$ is A-invariant, that is,

$$
A\left(\mathrm{Cycl}_{F}(A)\right) \subseteq \mathrm{Cycl}_{F}(A) .
$$

8. Corollary. The set $\operatorname{Cycl}\left(D_{0, g_{0}}\right)$ is $D_{0, g_{0}}$-invariant. 
Proof. The operator $D_{0, g_{0}}: E \rightarrow E$ is surjective. In fact, for $f \in E$ the function $h(z)=z f(z), z \in \mathbb{C}$, belongs to $E$ and $D_{0, g_{0}}(h)=f$. By Lemma $7 D_{0, g_{0}}\left(\operatorname{Cycl}\left(D_{0, g_{0}}\right)\right) \subseteq$ $\operatorname{Cycl}\left(D_{0, g_{0}}\right)$.

Put for $z \in \mathbb{C}, f \in E$

$$
\widetilde{T}_{z}(f)(t):=\left\{\begin{array}{cl}
\frac{f(t) g_{0}(z)-f(z) g_{0}(t)}{t-z}, & t \neq z, \\
f^{\prime}(z) g_{0}(z)-f(z) g_{0}^{\prime}(z), & t \neq z,
\end{array}\right.
$$

Clearly, $\tilde{T}_{z} \in \mathcal{L}(E)$ for all $z \in \mathbb{C}$. Note that Krasichkov-Ternovskii [12, $\S 10$ ] used similar constructions in some spaces of entire functions of exponential type for the solution of a problem on the extension of spectral synthesis.

9. Theorem. The following assertions are equivalent:

(i) $f \in \operatorname{Cycl}\left(D_{0, g_{0}}\right)$.

(ii) The system $\left\{T_{z}(f): z \in \mathbb{C}\right\}$ is complete in $E$.

(iii) $f \notin \operatorname{Ker} B$ for each nonzero operator $B \in \mathcal{K}\left(D_{0, g_{0}}\right)$.

(iv) The system $\left\{\widetilde{T}_{z}(f): z \in \mathbb{C}\right\}$ is complete in $E$.

Proof. By Theorem 5 assertions ( $i i)$ and (iii) are equivalent.

$(i) \Rightarrow($ iii $)$ : Let $B \in \mathcal{K}\left(D_{0, g_{0}}\right)$ be nonzero. By Theorem 5 there exists $\varphi \in E^{\prime} \backslash\{0\}$ such that $B(h)(z):=l\left(T_{z}(h)\right)$ for all $h \in E$ and $z \in \mathbb{C}$. We assume that $f \in \operatorname{Ker} B$. Then for all $n \geq 0$

$$
0=D_{0}^{n}(B(f))=B\left(D_{0}^{n}(f)\right) .
$$

Hence $D_{0}^{n}(f) \in \operatorname{Ker} B$ for each $n \geq 0$ and $\operatorname{span}\left\{D_{0}^{n}(f): n \geq 0\right\} \subseteq \operatorname{Ker} B$. Since $f$ is a cyclic vector for $D_{0, g_{0}}$, the closed subspace Ker $B$ of $E$ coincides with $E$ and consequently $B$ is zero operator. A contradiction.

$(i i) \Rightarrow(i)$ : We take $l \in E^{\prime}$ such that $l\left(D_{0, g_{0}}^{n}(f)\right)=0$ for each $n \geq 0$. Let $\varphi_{n}, n \geq 0$, be the functionals defined by Lemma 1. Since the system $\left\{\varphi_{n}: n \geq 0\right\}$ is complete in $\left(E^{\prime}, \tau\right)$, there exists a net $\left\{\Phi_{\alpha}=\sum_{j=0}^{m_{\alpha}} a_{j \alpha} \varphi_{j}: \alpha \in \Lambda\right\}$ convergent to $l$ in $\left(E^{\prime}, \tau\right)$.

Let as before $\widetilde{f}(t, z):=T_{z}(f)(t), f \in E, t, z \in \mathbb{C}$. By Lemma 1

$$
D_{0, g_{0}}^{n}(f)(z)=\left(\varphi_{n}\right)_{t}(\tilde{f}(t, z)), z \in \mathbb{C}, n \geq 0 .
$$

Fix $n \geq 0$. Taking into account Lemma 3 and Lemma 4, we have:

$$
\begin{gathered}
0=l\left(D_{0, g_{0}}^{n}(f)\right)=l_{z}\left(\left(\varphi_{n}\right)_{t}(\tilde{f}(t, z))\right)=\lim _{\alpha \in \Lambda}\left(\Phi_{\alpha}\right)_{z}\left(\left(\varphi_{n}\right)_{t}(\tilde{f}(t, z))\right)= \\
\lim _{\alpha \in \Lambda} \sum_{j=0}^{m_{\alpha}} a_{j \alpha}\left(\varphi_{j}\right)_{z}\left(\left(\varphi_{n}\right)_{t}(\tilde{f}(t, z))\right)=\lim _{\alpha \in \Lambda} \sum_{j=0}^{m_{\alpha}} a_{j \alpha}\left(\varphi_{n}\right)_{t}\left(\left(\varphi_{j}\right)_{z}(\widetilde{f}(t, z))\right)= \\
\lim _{\alpha \in \Lambda}\left(\varphi_{n}\right)_{t}\left(\sum_{j=0}^{m_{\alpha}} a_{j \alpha}\left(\varphi_{j}\right)_{z}(\tilde{f}(t, z))\right)=\lim _{\alpha \in \Lambda}\left(\varphi_{n}\right)_{t}\left(\left(\Phi_{\alpha}\right)_{z}(\tilde{f}(t, z))=\right. \\
\left(\varphi_{n}\right)_{t}\left(l_{z}(\tilde{f}(t, z))\right)=\left(\varphi_{n}\right)_{t}\left(l_{z}\left(T_{z}(f)(t)\right)\right)= \\
\left(\varphi_{n}\right)_{t}\left(l_{z}\left(T_{t}(f)(z)\right)\right)=\left(\varphi_{n}\right)_{t}\left(l\left(T_{t}(f)\right)\right) .
\end{gathered}
$$


Hence for the entire function $h(t):=l\left(T_{t}(f)\right)$ depending on $t$ we have $h^{(n)}(0)=0$ for all $n \geq 0$. Therefore $l\left(T_{t}(f)\right)=0$ for all $t \in \mathbb{C}$, that, by hypothesis, implies that $l=0$. Consequently, (i) holds.

$(i i) \Rightarrow(i v)$ : Since for $t \neq z$

$$
g_{0}(z) f(t)-\frac{t f(t) g_{0}(z)-z f(z) g_{0}(t)}{t-z}=-z \frac{f(t) g_{0}(z)-f(z) g_{0}(t)}{t-z},
$$

the equality

$$
g_{0}(z) f-T_{z}(f)=-z \widetilde{T}_{z}(f), z \in \mathbb{C},
$$

holds. Fix $l \in E^{\prime}$ such that $l\left(\widetilde{T}_{z}(f)\right)=0$ for all $z \in \mathbb{C}$. Acting by $l$ on the equality (2), we have for all $z \in \mathbb{C}$

$$
g_{0}(z) l(f)=l\left(T_{z}(f)\right)
$$

We put for $h \in E$ and $z \in \mathbb{C}$

$$
B(h)(z):=l\left(T_{z}(h)\right) .
$$

By Theorem $5, B \in \mathcal{K}\left(D_{0, g_{0}}\right)$. Hence for all $t \in \mathbb{C}$, since $D_{0, g_{0}}\left(g_{0}\right)=0$,

$$
0=D_{0, g_{0}}\left(l(f) g_{0}\right)(t)=D_{0, g_{0}}(B(f))(t)=B\left(D_{0, g_{0}}(f)\right)(t)=l\left(T_{t}\left(D_{0, g_{0}}(f)\right)\right) .
$$

By $(i i) \Rightarrow(i)$ the function $f$ is a cyclic vector for $D_{0, g_{0}}$ and, by Corollary $8, D_{0, g_{0}}(f) \in$ $\operatorname{Cycl}\left(D_{0, g_{0}}\right)$. Consequently, by $(i) \Rightarrow(i i), l=0$. Therefore (iv) satisfies.

$(i v) \Rightarrow(i i)$ : Fix $l \in E^{\prime}$ such that $l\left(T_{z}(f)\right)=0$ for all $z \in \mathbb{C}$. If we act by $l$ on the equality (2), we have for all $z \in \mathbb{C}$

$$
g_{0}(z) l(f)=-z l\left(\widetilde{T}_{z}(f)\right)
$$

that implies, since $g_{0}(0)=1$, that $l(f)=0$. Consequently, the entire function $l\left(\widetilde{T}_{z}(f)\right)$ depending on $z$ vanishes on $\mathbb{C}$. By hypothesis, $l=0$. Hence (ii) is satisfied.

By $M$ we denote the operator of multiplication by the independent variable:

$$
M(f)(z):=z f(z), f \in E, z \in \mathbb{C} .
$$

It follows from $(1)$ that $M \in \mathcal{L}(E)$. Denote by $I$ the identity mapping.

10. Lemma. Assume that $g_{0}(\alpha) \neq 0$ for some $\alpha \in \mathbb{C}$. Let $f \in E$. Consider assertions

(i) The system $\left\{T_{z}(f): z \in \mathbb{C}\right\}$ is complete in $E$.

(ii) The system $\left\{T_{z}((M-\alpha I)(f)): z \in \mathbb{C}\right\}$ is complete in $E$.

Then $(i) \Rightarrow(i i)$.

Proof. We use the equality

$$
\begin{gathered}
t g_{0}(z) f(t)-\frac{t(t-\alpha) f(t) g_{0}(z)-z(z-\alpha) f(z) g_{0}(t)}{t-z}= \\
-(z-\alpha) \frac{t f(t) g_{0}(z)-z f(z) g_{0}(t)}{t-z}, t \neq z .
\end{gathered}
$$

This implies that for all $z \in \mathbb{C}$

$$
g_{0}(z) M(f)-T_{z}((M-\alpha I)(f))=-(z-\alpha) T_{z}(f) .
$$


Assume that $(i)$ holds. We take $l \in E^{\prime}$ such that $l\left(T_{z}((M-\alpha I)(f))\right)=0$ for all $z \in \mathbb{C}$. Acting by $l$ on the equality (3), we have for all $z \in \mathbb{C}$

$$
g_{0}(z) l(M(f))=-(z-\alpha) l\left(T_{z}(f)\right) .
$$

From $g_{0}(\alpha) \neq 0$ it follows that $l(M(f))=0$. Since $l\left(T_{z}(f)\right)$ is an entire function depending on $z$, then $l\left(T_{z}(f)\right)=0$ for all $z \in \mathbb{C}$. By hypothesis, $l=0$. Hence (ii) satisfies.

11. Remark. If $g_{0}(\alpha)=0$ and $f(\alpha)=0$ for some $\alpha \in \mathbb{C}$ and some $f \in E$, then $D_{0, g_{0}}^{n}(f)(\alpha)=0$ for all $n \geq 0$.

12. Corollary. (i) Let $f \in \operatorname{Cycl}\left(D_{0, g_{0}}\right)$ and $P$ be a polynomial. Then $P f \in \operatorname{Cycl}\left(D_{0, g_{0}}\right)$ if and only if $P$ does not have common zeros with $g_{0}$.

(ii) Assume that the function $g_{0}$ has no zeros in $\mathbb{C}$. Then $P f \in \operatorname{Cycl}\left(D_{0, g_{0}}\right)$ for each $f \in \operatorname{Cycl}\left(D_{0, g_{0}}\right)$ and for each nonzero polynomial $P$.

Proof. (i): Let $P f \in \operatorname{Cycl}\left(D_{0, g_{0}}\right)$. We assume that $P$ and $g_{0}$ have a common zero $\alpha \in \mathbb{C}$. Then $D_{0, g_{0}}^{n}(P f)(\alpha)=0$ for all $n \geq 0$. Therefore each function in $F:=\operatorname{span}\left\{D_{0, g_{0}}^{n}(P f)\right.$ : $n \geq 0\}$ and in the closure $F$ in $E$, which coincides with $E$, vanishes at the point $\alpha$. A contradiction.

If $P$ and $g_{0}$ do not have common zeros the function $P f$ is a cyclic vector by Lemma 10 .

(ii) follows from (i).

For $z \in \mathbb{C}$ the Pommiez operators $D_{z}$ are defined by

$$
D_{z}(f)(t):=\left\{\begin{array}{cl}
\frac{f(t)-f(z)}{t-z}, & t \neq z, \\
f^{\prime}(z), & t=z,
\end{array}\right.
$$

for $f \in H(\mathbb{C})$. If $g_{0} \equiv 1 \in E$, all operators $D_{z}$ map continuously and linearly $E$ into itself.

13. Corollary. Let $g_{0} \equiv 1, f \in E$. The following statements are equivalent:

(i) $f \in \operatorname{Cycl}\left(D_{0}\right)$.

(ii) The system $\left\{T_{z}(f): z \in \mathbb{C}\right\}$ is complete in $E$.

(iii) The system $\left\{D_{z}(f): z \in \mathbb{C}\right\}$ is complete in $E$.

It follows from Theorem 9 , since $D_{z}(f)=\widetilde{T}_{z}(f)$ if $g_{0} \equiv 1$.

Further we will use essentially the following relation between operators $\tilde{T}_{z}$ and $D_{z}$.

14. Lemma. For all $f \in E, z \in \mathbb{C}$

$$
\tilde{T}_{z}(f)=g_{0}(z) D_{z}(f)-f(z) D_{z}\left(g_{0}\right) .
$$
E.)

(We note that $\tilde{T}_{z}(f) \in E$, but $g_{0}(z) D_{z}(f)$ and $f(z) D_{z}\left(g_{0}\right)$ can need not belong to

This assertion can be verified directly. 


\section{A CONCRETE EXAMPLE}

Let $Q$ be a convex locally closed set in $\mathbb{C}$, that is, a convex set having a countable fundamental sequence of compact subsets (see [18], [2]). By [18, Lemma 1.2] $Q$ is the union of the relative interior of $Q$ and an open portion of the relative boundary of $Q$. The family of such sets $Q$ contains all convex domains in $\mathbb{C}$ and all convex compact sets in $\mathbb{C}$. We assume that $0 \in Q$. Let $\left(Q_{n}\right)_{n \in \mathbb{N}}$ be an increasing fundamental sequence of compact sets in $Q$. Without loss of generality all sets $Q_{n}$ are convex and $0 \in Q_{1}$. We consider the spaces $H\left(Q_{n}\right)$ of all functions which are holomorphic on some neighborhood of $Q_{n}$ with their natural inductive topology. Let $H(Q)$ be the vector space of all holomorphic functions on $Q$, that is, holomorphic on some open neighborhood of $Q$. Since the algebraic equality $H(Q)=\bigcap_{n \in \mathbb{N}} H\left(Q_{n}\right)$ holds we endow $H(Q)$ with the projective topology of $H(Q):=\operatorname{proj}_{\leftarrow n} H\left(Q_{n}\right)$.

By $H_{\Omega}(z):=\sup _{t \in \Omega} \operatorname{Re}(t z), z \in \mathbb{C}$, we denote the support function of a set $\Omega \subset \mathbb{C}$. Put

$$
v_{n, k}(z):=H_{Q_{n}}(z)+|z| / k, z \in \mathbb{C}, n, k \in \mathbb{N} .
$$

All functions $v_{n, k}$ are subadditive and positively homogeneous of degree 1 . The sequence $\left(v_{n, k}\right)_{n, k \in \mathbb{N}}$ satisfies the condition (1).

We put $E_{Q}:=E$. Note that for each $n \in \mathbb{N}$ an entire function $h$ of exponential type belongs to $E_{n}$ if and only if the conjugate diagram of $h$ is contained in $Q_{n}$.

By [18, Lemma 1.10] the Laplace transform

$$
\mathcal{F}(\varphi)(z):=\varphi\left(e^{\cdot z}\right), \varphi \in H(Q)^{\prime}, z \in \mathbb{C},
$$

is a topological isomorphism of the strong dual of $H(Q)$ onto the space $E_{Q}$. We will describe cyclic vectors for an operator $D_{0, g_{0}}$ in $E_{Q}$.

3.1. An interpolating function. Let $K$ be a convex compact set in $\mathbb{C}$ such that $0 \in K$. For an entire function $f$ of exponential type with the conjugate diagram contained in $K$, for $x \in H(K)$ Leont'ev (see [13]) introduced the interpolating function $\omega_{f}: \mathbb{C} \times H(K) \rightarrow \mathbb{C}$ by

$$
\omega_{f}(z, x):=\frac{1}{2 \pi i} \int_{C}\left(\int_{0}^{t} x(t-\xi) e^{z \xi} d \xi\right) \gamma_{f}(t) d t, z \in \mathbb{C}, x \in H(K) .
$$

Here $C$ is a closed convex curve which surrounds $K$, the function $x$ is holomorphic on the closure of the interior of the curve $C, \gamma_{f}$ is the Borel transform of $f$. The inner integral is taken along the segment $[0, t]$. Note that $\omega_{f}(z, x)$ is the entire function depending on $z$.

By [13, Theorem 1] the following uniqueness theorem holds.

15. Theorem. Let $K$ be a convex compact set in $\mathbb{C}$ containing $0, f$ be an entire function of exponential type with the conjugate diagram contained in $K$. If $f$ has infinitely many zeros and for $x \in H(K)$ the function $\omega_{f}(z, x) / f(z)$ is entire one depending on $z$, then $x=0$.

Let $e_{\nu}(z):=e^{\nu z}, \quad z, \nu \in \mathbb{C}$. 
16. Remark. (a) Put

$$
\langle x, h\rangle:=\mathcal{F}^{-1}(h)(x), x \in H(K), h \in E_{K} .
$$

By [6, Example 1]

$$
\left\langle x, D_{z}(f)\right\rangle=\omega_{f}(z, x), x \in H(K), f \in E_{K}, z \in \mathbb{C} .
$$

(b) For each $\nu \in K, j \geq 0$, for the function $h(z):=z^{j} e^{\nu z}$

$$
\gamma_{h}(t)=j !(t-\nu)^{-j-1} \text {. }
$$

(c) The function $e_{\nu}$ belongs to $E_{Q}$ if and only if $\nu \in Q$.

(d) For each $\nu \in Q, x \in H(Q), z \in \mathbb{C}$

$$
\omega_{e_{\nu}}(z, x)=\int_{0}^{\nu} x(\nu-\xi) e^{z \xi} d \xi=e^{\nu z} \int_{0}^{\nu} x(\eta) e^{-z \eta} d \eta .
$$

\subsection{Cyclic elements of the Pommiez operator in $E_{Q}$.}

17. Remark. (a) Since $0 \in Q$, the function $h \equiv 1$ belongs to $E_{Q}$. Hence $D_{z} \in \mathcal{L}\left(E_{Q}\right)$ for each $z \in \mathbb{C}$.

(b) For each function $h \in E_{Q}$ the following alternative is valid: either $h$ has infinitely many zeros or there exist $\lambda \in Q$ and a polynomial $P$ such that $h=P e_{\lambda}$.

18. Lemma. Let $y$ be a continuous function on a segment $[\alpha, \beta](\alpha \neq \beta)$ and assume that the entire function $U(z)=e^{\alpha z} \int_{\alpha}^{\beta} y(\eta) e^{-z \eta} d \eta$ is a polynomial. Then $y=0$ on $[\alpha, \beta]$.

Proof. We note that

$$
U(z)=e^{\alpha z} \int_{\alpha}^{\beta} y(\eta) e^{-z \eta} d \eta=\int_{\alpha}^{\beta} y(\eta) e^{z(\alpha-\eta)} d \eta=-\int_{0}^{\alpha-\beta} y(\alpha-\xi) e^{z \xi} d \xi .
$$

Let $\alpha-\beta=\rho e^{i \varphi}$ with $\rho>0$ and $\varphi \in \mathbb{R}$. We make the change of the variable $\xi=r e^{i \varphi}$, where $r \in[0, \rho]$, and we take $z$ in the form $z:=-t e^{-i \varphi}$ with $t>0$. Then

$$
U(z)=-\int_{0}^{\rho} y\left(\alpha-r e^{i \varphi}\right) e^{-t r} e^{i \varphi} d r
$$

Hence there exists $D>0$ such that

$$
|U(z)| \leq D \int_{0}^{\rho} e^{-t r} d r=-\frac{D}{t}\left(e^{-\rho t}-1\right)
$$

From here it follows that $|U(z)| \rightarrow 0$ as $t \rightarrow+\infty$. Since $U$ is a polynomial, $U=0$ on $\mathbb{C}$. From the completeness of the system $\left\{e_{z}: z \in \mathbb{C}\right\}$ in the space of all continuous functions on the segment $[0, \rho]$ it follows that the function $y$ vanishes on $[\alpha, \beta]$.

19. Theorem. (I) Suppose that the function $g_{0}$ has infinitely many zeros. The following assertions are equivalent: 
(i) $f \in \operatorname{Cycl}\left(D_{0, g_{0}}\right)$.

(ii) Functions $f$ and $g_{0}$ do not have common zeros.

(II) Suppose that $g_{0}=P e_{\lambda}$ for some $\lambda \in Q$ and for some polynomial $P$ with $P(0)=$ 1. The following assertions are equivalent:

(i) $f \in \operatorname{Cycl}\left(D_{0, g_{0}}\right)$.

(iii) Functions $f$ and $g_{0}$ do not have common zeros and $f$ is not a function of the form $R e_{\lambda}$ where $R$ is a polynomial.

Proof. (I) $(i) \Rightarrow($ ii $)$ : We assume that $f$ and $g_{0}$ have a common zero $\alpha \in \mathbb{C}$. Proceeding as in the proof of (i) of Corollary 12, we conclude that each function in $E$ vanishes in $\alpha$. A contradiction.

$($ ii $) \Rightarrow(i)$ : We take $n \in \mathbb{N}$ such that $g_{0} \in E_{n}$. The conjugate diagram of $g_{0}$ is contained in $Q_{n}$. Fix $x \in H(Q) \subset H\left(Q_{n}\right)$ such that $\left\langle x, \tilde{T}_{z}(f)\right\rangle=0$ for all $z \in \mathbb{C}$. By Lemma 14 for each $z \in \mathbb{C}$

$$
g_{0}(z)\left\langle x, D_{z}(f)\right\rangle=f(z)\left\langle x, D_{z}\left(g_{0}\right)\right\rangle .
$$

By Remark 16 (a) for all $z \in \mathbb{C}$

$$
g_{0}(z) \omega_{f}(z, x)=f(z) \omega_{g_{0}}(z, x) .
$$

Since $g_{0}$ and $f$ do not have common zeros, $\omega_{g_{0}}(z, x) / g_{0}(z)$ is entire function depending on $z$. By Theorem $15, x=0$. Consequently, the system $\left\{\tilde{T}_{z}(f): z \in \mathbb{C}\right\}$ is complete in $E_{Q}$. By Theorem $9, f \in \operatorname{Cycl}\left(D_{0, g_{0}}\right)$.

(II) $($ iii $) \Rightarrow(i)$ : Consider all possible situations.

Suppose that $f$ has infinitely many zeros. Proceeding as in the proof of the implication $(i i) \Rightarrow(i)$, we obtain that $f \in \operatorname{Cycl}\left(D_{0, g_{0}}\right)$.

Suppose now that $f$ has no zeros or $f$ has finite many zeros. Then $f=S e_{\mu}$ for some $\mu \in Q$ such that $\mu \neq \lambda$ and for some polynomial $S$. We fix $n \in \mathbb{N}$ such that $\lambda, \mu \in Q_{n}$.

Let at first $S \equiv$ const $=C_{0} \neq 0$. Without loss of generality $C_{0}=1$. Let $P(z)=$ $\sum_{j=0}^{m} a_{j} z^{j}$ where $m \in \mathbb{N}$ and $a_{0}=1$. We fix $x \in H(Q)$ with $\left\langle x, \tilde{T}_{z}(f)\right\rangle=0$ for all $z \in \mathbb{C}$.

By Lemma 14 and Remark 16 (a) for all $z \in \mathbb{C}$

$$
g_{0}(z) \omega_{f}(z, x)=f(z) \omega_{g_{0}}(z, x) .
$$

Let $m=1$. By Remark $16(\mathrm{~d})$

$$
\omega_{f}(z, x)=e^{\mu z} Y(\mu, z)
$$

where $Y(t, z):=\int_{0}^{t} x(\eta) e^{-z \eta} d \eta$. Hence

$$
g_{0}(z) Y(\mu, z)=\omega_{g_{0}}(z, x), z \in \mathbb{C} .
$$

By Remark 16 (b) for $K:=Q_{n}$, for some curve $C$ as in 3.1

$$
\omega_{g_{0}}(z, x)=e^{z \lambda} Y(\lambda, z)+a_{1} \frac{1}{2 \pi i} \int_{C} \frac{1}{(t-\lambda)^{2}}\left(\int_{0}^{t} x(t-\xi) e^{z \xi} d \xi\right) d t .
$$


Since

$$
\int_{0}^{t} x(t-\xi) e^{z \xi} d \xi=e^{z t} \int_{0}^{t} x(\eta) e^{-z \eta} d \eta
$$

we have by the integral Cauchy formula

$$
\begin{gathered}
\omega_{g_{0}}(z, x)=e^{z \lambda} Y(\lambda, z)+\left.a_{1} \frac{d}{d t}\left(e^{z t} \int_{0}^{t} x(\eta) e^{-z \eta} d \eta\right)\right|_{t=\lambda}= \\
e^{z \lambda} Y(\lambda, z)+a_{1}\left(z e^{\lambda z} Y(\lambda, z)+x(\lambda)\right) .
\end{gathered}
$$

Thus by (4)

$$
e^{\lambda z}\left(1+a_{1} z\right) Y(\mu, z)=e^{z \lambda} Y(\lambda, z)+a_{1}\left(z e^{\lambda z} Y(\lambda, z)+x(\lambda)\right) .
$$

From here it follows that for all $z \in \mathbb{C}$

$$
e^{\lambda z}\left(1+a_{1} z\right) \int_{\lambda}^{\mu} x(\eta) e^{-z \eta} d \eta=a_{1} x(\lambda) .
$$

By Lemma 18, $x=0$ on $[\lambda, \mu]$ and consequently $x=0$ as an element of $H(Q)$.

Let now $m \geq 2$. Then by Remark 16 (b) and by the integral Cauchy formula

$$
\begin{gathered}
\omega_{g_{0}}(z, x)=e^{\lambda z} Y(\lambda, z)+\sum_{j=1}^{m} a_{j} \frac{j !}{2 \pi i} \int_{C} \frac{d t}{(t-\lambda)^{j+1}} \int_{0}^{t} x(t-\xi) e^{z \xi} d \xi= \\
e^{\lambda z} Y(\lambda, z)+\left.\sum_{j=1}^{m} a_{j} \frac{d^{j}}{d t^{j}}\left(e^{z t} \int_{0}^{t} x(\eta) e^{-z \eta} d \eta\right)\right|_{t=\lambda}= \\
e^{\lambda z} Y(\lambda, z)+a_{1}\left(z e^{\lambda z} Y(\lambda, z)+x(\lambda)\right)+\left.\sum_{j=2}^{m} a_{j} \frac{d^{j}}{d t^{j}}\left(e^{z t} \int_{0}^{t} x(\eta) e^{-z \eta} d \eta\right)\right|_{t=\lambda} .
\end{gathered}
$$

For $j \geq 2$

$$
\begin{gathered}
\frac{d^{j}}{d t^{j}}\left(e^{z t} \int_{0}^{t} x(\eta) e^{-z \eta} d \eta\right)=\sum_{s=0}^{j} C_{j}^{s} z^{j-s} e^{z t} \frac{d^{s}}{d t^{s}}(Y(t, z))=z^{j} e^{z t} Y(t, z)+ \\
C_{j}^{1} z^{j-1} e^{z t} x(t) e^{-z t}+\sum_{s=2}^{j} C_{j}^{s} z^{j-s} e^{z t} \sum_{r=0}^{s-1} C_{s-1}^{r}(-1)^{r} z^{r} e^{-z t} x^{(s-1-r)}(t) .
\end{gathered}
$$

Consequently,

$$
\begin{gathered}
\omega_{g_{0}}(z, x)=e^{\lambda z} Y(\lambda, z)+a_{1}\left(z e^{\lambda z} Y(\lambda, z)+x(\lambda)\right)+ \\
\sum_{j=2}^{m} a_{j}\left(z^{j} e^{\lambda z} Y(\lambda, z)+\right. \\
\left.C_{j}^{1} z^{j-1} x(\lambda)+\sum_{s=2}^{j} C_{j}^{s} z^{j-s} e^{\lambda z} \sum_{r=0}^{s-1} C_{s-1}^{r}(-1)^{r} z^{r} e^{-\lambda z} x^{(s-1-r)}(\lambda)\right)=
\end{gathered}
$$




$$
\begin{gathered}
e^{\lambda z} Y(\lambda, z)+a_{1} z e^{\lambda z} Y(\lambda, z)+\sum_{j=2}^{m} a_{j} z^{j} e^{\lambda z} Y(\lambda, z)+W(z)= \\
e^{\lambda z} Y(\lambda, z) g_{0}(z)+W(z)
\end{gathered}
$$

where $W$ is a polynomial depending on $z$. It has the form

$$
W(z)=\sum_{p=0}^{m-1} w_{p}(z) x^{(p)}(\lambda)
$$

where polynomials $w_{p}$ do not depend on $x \in H(Q)$. Hence by (4) for all $z \in \mathbb{C}$

$$
e^{\lambda z}\left(1+\sum_{j=1}^{m} a_{j} z^{j}\right) \int_{\lambda}^{\mu} x(\eta) e^{-z \eta} d \eta=W(z) .
$$

From here it follows that the entire function $e^{\lambda z} \int_{\lambda}^{\mu} x(\eta) e^{-z \eta} d \eta$ depending on $z$ is a polynomial. By Lemma $18 x=0$. Thus the system $\left\{\tilde{T}_{z}: z \in \mathbb{C}\right\}$ is complete in $E_{Q}$ and by Theorem $9 f \in \operatorname{Cycl}\left(D_{0, g_{0}}\right)$.

Finally, for arbitrary polynomial $S$ which does not have common zeros with $P$ the assertion follows from Corollary 12 (i).

$(i) \Rightarrow($ iii $)$ : From Remark 11 it follows that $f$ and $g_{0}$ do not have common zeros. We assume that there exists a polynomial $R$ of degree at most $s \in \mathbb{N}$ such that $f=R e_{\lambda}$. Let $P(z):=\sum_{j=0}^{m} a_{j} z^{j}, m \in \mathbb{N}$. As in the proof of the implication $(i i i) \Rightarrow(i)$ we conclude that there are polynomials $w_{p}, v_{q}$ such that for all $x \in H(Q)$

$$
\omega_{g_{0}}(z, x)=P(z) e^{\lambda z} Y(\lambda, z)+W(z)
$$

and

$$
\omega_{f}(z, x)=R(z) e^{\lambda z} Y(\lambda, z)+V(z)
$$

where $W(z)=\sum_{p=0}^{m-1} x^{(p)}(\lambda) w_{p}(z)$ and $V(z)=\sum_{q=0}^{s-1} x^{(q)}(\lambda) v_{q}(z)$. There exists a nonzero function $x \in H(Q)$ such that $x^{(r)}(\lambda)=0$ if $0 \leq r \leq \max (m, s)$. Then $W=V=0$ and for all $z \in \mathbb{C}$

$$
P(z) e^{\lambda z}\left(R(z) e^{\lambda z} Y(\lambda, z)+V(z)\right)=R(z) e^{\lambda z}\left(P(z) e^{\lambda z} Y(\lambda, z)+W(z)\right),
$$

that is, $g_{0}(z) \omega_{f}(z, x)=f(z) \omega_{g_{0}}(z, x)$ for all $z \in \mathbb{C}$. Consequently, by Remark 16 (a)

$$
g_{0}(z)\left\langle x, D_{z}(f)\right\rangle=f(z)\left\langle x, D_{z}\left(g_{0}\right)\right\rangle
$$

and, by Lemma $14,\left\langle x, \tilde{T}_{z}(f)\right\rangle=0$ for all $z \in \mathbb{C}$. Hence the system $\left\{\tilde{T}_{z}(f): z \in \mathbb{C}\right\}$ is incomplete in $E$ and by Theorem $9 f \notin \operatorname{Cycl}\left(D_{0, g_{0}}\right)$. A contradiction. 
3.3. Invariant subspaces of the Pommiez operator in $E_{Q}$. We apply Theorem 19 to a description of proper closed $D_{0, g_{0}}$-invariant subspaces of $E_{Q}$ where $g_{0}$ has no zeros.

Denote by $\mathbb{C}[z]$ (resp. $\mathbb{C}[z]_{n}$ for integer $n \geq 0$ ) the space of all polynomials (resp. of degree at most $n)$. Put for integer $n \geq 0$ and $\lambda \in \mathbb{C}$

$$
\mathcal{P}_{n}\left(e_{\lambda}\right):=e_{\lambda} \cdot \mathbb{C}[z]_{n}:=\left\{e_{\lambda} P: P \in C[z]_{n}\right\} .
$$

20. Corollary. Let $g_{0}=e_{\lambda}$ for some $\lambda \in Q$.

(i) For each integer $n \geq 0$ the space $\mathcal{P}_{n}\left(e_{\lambda}\right)$ is a proper closed $D_{0, g_{0}}$-invariant subspace of $E_{Q}$.

(ii) For each proper closed $D_{0, g_{0}}$-invariant subspace $\mathcal{P}$ of $E_{Q}$ there is $n \geq 0$ such that $\mathcal{P}=\mathcal{P}_{n}\left(e_{\lambda}\right)$.

Proof. (i): Each space $\mathcal{P}_{n}\left(e_{\lambda}\right)$ is finite-dimensional and hence closed. Clearly $\mathcal{P}_{n}\left(e_{\lambda}\right) \neq$ $E_{Q}$ and $\mathcal{P}_{n}\left(e_{\lambda}\right)$ is $D_{0, g_{0}}$-invariant.

(ii): Let $\mathcal{P}$ be a proper closed $D_{0, g_{0}}$-invariant subspace of $E_{Q}$. By Theorem 19 $\mathcal{P} \subset e_{\lambda} \cdot \mathbb{C}[z]$. Show that

$$
n(\mathcal{P}):=\sup \left\{\operatorname{deg}(P): P e_{\lambda} \in \mathcal{P}\right\}<+\infty .
$$

We assume that $n(\mathcal{P})=+\infty$. Note that for each polynom $S$ of degree $m \in \mathbb{N}$, for the function $h=S e_{\lambda}$ the function $D_{0, g_{0}}(h)$ has the form $S_{1} e_{\lambda}$ where $S_{1}$ is a polynomial of degree $m-1$. Hence for each $m \geq 0$ there exists a polynomial $R_{m}$ such that $\operatorname{deg}\left(R_{m}\right)=m$ and $R_{m} e_{\lambda} \in \mathcal{P}$. From here it follows that $e_{\lambda} \cdot \mathbb{C}[z] \subset \mathcal{P}$.

Let $\lambda \in Q_{n}$. We take $p \in \mathbb{N}$ and $h \in E_{p}$. By [11, Theorem 4.4] there are polynomials $R_{j}, j \in \mathbb{N}$, such that $R_{j} e_{\lambda} \rightarrow h$ in $E_{q}$ where $q=\max (n, p)$. Hence the subspace $e_{\lambda} \cdot \mathbb{C}[z]$ is dense in $E_{Q}$. Consequently, $\mathcal{P}=E_{Q}$. A contradiction. Thus $n(\mathcal{P})<+\infty$ and $\mathcal{P}=\mathcal{P}_{n(\mathcal{P})}\left(e_{\lambda}\right)$.

3.4. Ideals in the algebra $(H(Q), *)$. Let $E$ be the space as in $\S 1$. It is possible to connect with the operator $D_{0, g_{0}}$ a multiplication in $E^{\prime}$. By [8] for all $\varphi, \psi \in E^{\prime}$ the multiplication (convolution) $\varphi \otimes \psi$ is defined by

$$
\varphi \otimes \psi(f):=\varphi_{z}\left(\psi\left(T_{z}(f)\right)\right), f \in E .
$$

The operation $\otimes$ is associative and commutative. The vector space $E^{\prime}$ with this multiplication is an algebra. For a set $T \subset E$ (resp. $T \subset E^{\prime}$ ) we denote by $T^{0}$ the polar of $T$ in $E^{\prime}$ (resp. in $E$ ). We endow $E^{\prime}$ with the Mackey topology $\tau\left(E^{\prime}, E\right)$. The following duality principle holds.

21. Lemma. The following assertions are equivalent:

(i) A set $L \subset E^{\prime}$ is a proper closed ideal in $\left(E^{\prime}, \otimes\right)$.

(ii) There exists a proper closed $D_{0, g_{0}}$-invariant subspace $\mathcal{P}$ of $E$ such that $L=\mathcal{P}^{0}$.

Proof. $(i) \Rightarrow(i i)$ : Let $L$ be a proper closed ideal in $\left(E^{\prime}, \otimes\right)$. Fix $f \in L^{0}$. Then $\psi \otimes \varphi(f)=0$ for all $\psi \in L$ and $\varphi \in E^{\prime}$. By Lemma $1 D_{0, g_{0}}(f)(z)=\varphi_{1}\left(T_{z}(f)\right)$ for all $z \in \mathbb{C}$. Hence for all $\psi \in L$

$$
\psi\left(D_{0, g_{0}}(f)\right)=\psi_{z}\left(\varphi_{1}\left(T_{z}(f)\right)\right)=\psi \otimes \varphi_{1}(f)=0 .
$$


Consequently, $D_{0, g_{0}}(f) \in L^{0}$, and $\mathcal{P}:=L^{0}$ is a proper closed $D_{0, g_{0}}$-invariant subspace of $E$. Since $L$ is a closed subspace of $E^{\prime}$, by bipolar theorem [17, Ch. III, 22.13] $L=L^{00}=\mathcal{P}^{0}$.

$($ ii $) \Rightarrow(i)$ : Let $L=\mathcal{P}^{0}$ for some proper closed $D_{0, g_{0}}$-invariant subspace $\mathcal{P}$ of $E$. Fix $\varphi \in E^{\prime}, \psi \in L$. By [8, the proof of Theorem 14] for all $z \in \mathbb{C}$ there exists a net $B_{\alpha, z}=\sum_{j=0}^{n_{\alpha, z}} b_{j, \alpha, z} D_{0, g_{0}}^{j}, \alpha \in \Lambda_{z}$, such that $\lim _{\alpha \in \Lambda_{z}} B_{\alpha, z}(f)=T_{z}(f)$ in $E$. Then for all $f \in \mathcal{P}$

$$
\begin{gathered}
\varphi \otimes \psi(f)=\varphi_{z}\left(\psi\left(T_{z}(f)\right)\right)=\varphi_{z}\left(\psi\left(\lim _{\alpha \in \Lambda_{z}} \sum_{j=0}^{n_{\alpha, z}} b_{j, \alpha, z} D_{0, g_{0}}^{j}(f)\right)\right)= \\
\varphi_{z}\left(\lim _{\alpha \in \Lambda_{z}} \sum_{j=0}^{n_{\alpha, z}} b_{j, \alpha, z} \psi\left(D_{0, g_{0}}^{j}(f)\right)\right)=0
\end{gathered}
$$

since $\psi\left(D_{0, g_{0}}^{j}(f)\right)=0$ for all $j \geq 0$. Consequently, $\varphi \otimes \psi \in \mathcal{P}^{0}$, and $L$ is a proper closed ideal in $\left(E^{\prime}, \otimes\right)$.

We return to the space $E_{Q}$. By [16, Proposition 1.9] the projective topology in $H(Q)$ coincides with the inductive topology of $\operatorname{ind}_{\Omega} H(\Omega)$ where $\Omega$ runs over all open neighborhoods $\Omega$ of $Q$ and where $H(\Omega)$ is the Fréchet space of all holomorphic functions on $\Omega$. By [16, $\S 3$, p. 65] $H(Q)$ is a Montel space. Hence [17, Remark 24.24 (a)] the space $H(Q)$ is reflexive, and $E_{Q}^{\prime}$ can be identified with the space $H(Q)$. In addition, the transform

$$
\mathcal{J}: E_{Q}^{\prime} \rightarrow H(Q), \varphi \mapsto \varphi\left(e_{z}\right),
$$

is a topological isomorphism of the strong dual of $E_{Q}$ onto $H(Q)$. Put $\widehat{\varphi}:=\mathcal{J}(\varphi)$, $\varphi \in E_{Q}^{\prime}$.

From [8, Example 3.2] it follows that for $g_{0} \equiv 1$ the multiplication $\otimes$ in $E_{Q}^{\prime}$ is realized in $H(Q)$ in the following way. For all $\varphi, \psi \in E_{Q}^{\prime}$

$$
\mathcal{J}(\varphi \otimes \psi)(z)=\widehat{\psi}(0) \widehat{\varphi}(z)+\int_{0}^{z} \widehat{\varphi}(\xi)(\widehat{\psi})^{\prime}(z-\xi) d \xi
$$

where the integral is taken along the segment $[0, z]$, and $z$ belongs to the union of some convex domains $G_{n}, n \in \mathbb{N}$, such that $Q_{n} \subset G_{n}$ and functions $\widehat{\varphi}, \widehat{\psi}$ are holomorphic on each $G_{n}$. Hence for $g_{0} \equiv 1$ the operation $\otimes$ is realized as the Duhamel product $*$ in $H(Q)$ :

$$
v * w(z)=w(0) v(z)+\int_{0}^{z} v(\xi) w^{\prime}(z-\xi) d \xi, v, w \in H(Q) .
$$

The space $H(Q)$ is an algebra with the multiplication $*$. We will describe proper closed ideals in $(H(Q), *)$. We note at first that for all $\lambda \in Q, h \in H(Q)$ and integer $n \geq 0$ the following equality holds:

$$
\mathcal{J}^{-1}(h)_{z}\left(z^{n} e^{\lambda z}\right)=h^{(n)}(\lambda)
$$

From Corollary 20 and Lemma 21 it follows 
22. Corollary. The following assertions are equivalent:

(i) $T$ is a proper closed ideal in $(H(Q), *)$.

(ii) There exists an integer $n \geq 0$ such that

$$
T=\left\{h \in H(Q) \mid h^{(j)}(0)=0,0 \leq j \leq n\right\} .
$$

Acknowledgement. The authors would like to express gratitude to Professor J. Bonet for fruitful discussions and valuable comments.

\section{REFERENCES}

[1] Binderman, Z.: Functional shifts induced by right invertible operators. Math. Nachr. 157, 211$224(1992)$

[2] Bonet, J., Meise, R., Melikhov, S. N.: The dual of the space of holomorphic functions on locally closed convex sets. Publ. Mat. 49, 487-509 (2005)

[3] Dimovski, I. N., Hristov, V. Z.: Commutants of the Pommiez operator. Int. J. Math. and Math. Science 8, 1239-1251 (2005)

[4] Douglas, R. G., Shapiro, H. S., Shields, A. I.: Cyclic vectors and invariant subspaces for the backward shift operator. Ann. Inst. Fourier (Grenoble) 20 (1), 37-76 (1970)

[5] Godefroy, G., Shapiro, J. H.: Operators with dense, invariant, cyclic vector manifolds. J. of Funct. Anal. 98, 229-269 (1991)

[6] Ivanova, O. A., Melikhov, S. N.: On A. F. Leont'ev's interpolating function. Ufa Mathematical Journal 6 (3), 17-27 (2014)

[7] Ivanova, O. A., Melikhov, S. N.: On the orbits of analytic functions with respect to a Pommiez type operator. Ufa Mathematical Journal 7 (4), 75-79 (2015)

[8] Ivanova, O. A., Melikhov, S. N.: On operators commuting with a Pommiez type operator in weighted spaces of entire functions. Algebra and Analysis 28 (2), 114-137 (2016)(in Russian). English translation in: St. Petersburg Math. J. 28 (2) (2017)

[9] Kaz'min, Yu. A.: On sequential remainders of a Taylor series. Vestnik MGU. Ser. 1, Matematika, Mekhanika 5, 35-46 (1963) (in Russian)

[10] Khaplanov, M. G.: On the completeness of some systems of analytic functions. Uchenye zapiski Rostovskogo gos. ped. instituta: Sb. statei. Rostov on Don 3, 53-58 (1955) (in Russian)

[11] Krasichkov-Ternovskii, I. F.: Invariant subspaces of analytic functions. I. Spectral analysis on the convex regions. Math. USSR-Sbornik 16, 471-500 (1972)

[12] Krasichkov-Ternovskii, I. F.: Invariant subspaces of analytic functions. III. On the extension of spectral synthesis. Math. USSR-Sbornik 17 (3), 327-348 (1972)

[13] Leont'ev, A. F.: On the uniqueness property. Math USSR-Sbornik 1 (2), 209-220 (1967)

[14] Linchuk, N. E.: Representation of commutants of a Pommiez operator and their applications. Math. Notes 44 (6), 926-930 (1988)

[15] Linchuk, Yu. S.: Cyclical elements of operators which are left-inverses to multiplication by an independent variable. Methods of Functional Analysis and Topology 12 (4), 384-388 (2006)

[16] Martineau, A.: Sur la topologie des espaces de functions holomorphes. Math. Ann. 163, 62-88 (1966).

[17] Meise, R., Vogt, D.: Introduction to Functional Analysis. The Clarendon Press, Oxford University Press, New York (1997)

[18] Melikhov, S. N., Momm, S.: Analytic solutions of convolution equations on convex sets with an obstacle in the boundary. Math. Scand. 86 (4), 293-319 (2000)

[19] Pommiez, M.: Sur les zéros des reste successifs des séries de Taylor, Acad. Sci. Univ. Toulouse 250 (7), 1168-1170 (1960)

[20] Pommiez, M.: Sur les restes successifs des séries de Taylor. C. R. Acad. Sci. 250 (15), 2669-2671 (1960)

[21] Pommiez, M.: Sur les restes et les dérivées des séries de Taylor. C. R. Acad. Sci. 251 (17), 1707-1709 (1960) 
[22] Pommiez, M.: Sur les différences divisées successives et les restes des séries de Newton généralisées. Ann. Fac. Sci. Univ. Toulouse 28, 101-110 (1964)

[23] Tkachenko, V. A.: Invariant subspaces and unicellularity of operators of generalized integration in spaces of analytic functionals. Math. Notes 22 (2), 613-618 (1977)

[24] Tkachenko, V. A.: Operators that commute with generalized integration in spaces of analytic functionals. Math. Notes 22 (2), 141-146 (1979) 